\title{
Primeira ocorrência de Lepidotes (Osteichthyes, Actinopterygii, Lepisosteiformes) na Formação São Sebastião, Berriasiano-Aptiano da Sub- bacia de Tucano Central, Bahia, Brasil
}

\author{
First occurrence of Lepidotes (Osteichthyes, Actinopterygii, \\ Lepisosteiformes) in the São Sebastião Formation, \\ Berriasian-Aptian of the Tucano Basin, Bahia, Brazil. \\ Hanna Carolina Lins de Paiva ${ }^{1 *}$, Rafael Costa da Silva ${ }^{2}$, \\ Caroline Couto Santos ${ }^{3}$, Carolina Reis ${ }^{3}$
}

RESUMO: Estudos recentes revelaram a ocorrência de peixes fósseis inéditos na Formação São Sebastiāo, Sub-bacia de Tucano Central, Nordeste do Brasil. O material consiste em escamas e ossos isolados, além de um exemplar em posição de articulação. Os exemplares foram identificados como "Lepidotes" sp., "Lepidotes" oliveirai e "Lepidotes" spp. (Actinopterygii, Lepisosteiformes) e estão preservados em arenitos originados de sistemas fluviais. A mistura de espécimes articulados e desarticulados permite sugerir que a associação fossilífera seria parautóctone ou mais provavelmente uma mistura de elementos autóctones e alóctones.

PALAVRAS-CHAVE: Nordeste do Brasil; Paleoictiofauna; Cretáceo Inferior; sistema fluvial.

\section{INTRODUÇÃO}

De acordo com Gallo-da-Silva (1998), Gallo (2000, 2005) e Gallo \& Brito (2004), o gênero Lepidotes (Agassiz, 1832) foi abundante em boa parte do mundo. Esses peixes atingiram diversos tamanhos e dominaram ambientes de água doce, salgada e salobra. Embora López-Arbarello (2012) tenha considerado como Lepidotes apenas os indivíduos do Neojurássico da Europa Central, muitos indivíduos desse gênero foram encontrados e registrados no Brasil, nas bacias sedimentares do Nordeste, e seus registros datam do Neojurássico ao Neocretáceo (Brito \& Gallo 2003a,b, Gallo $\&$ Brito 2004, Santos \& Carvalho 2009).
ABSTRACT: Recent studies revealed the first occurrence of fossil fish in the São Sebastiāo Formation, Tucano Basin, Northeastern Brazil. The material consists of isolated bones and scales, as well as an articulated specimen preserved in fluvial sandstones. The fish remains have been identified as "Lepidotes" sp., " $L$ ". oliveirai and "L". spp. (Actinopterygii, Lepisosteiformes). The presence of both articulated and disarticulated specimens suggests that the fossiliferous association could be parautochthonous or probably a mix of autochthonous and allochthonous elements.

KEYWORDS: Northeastern Brazil; Palaeoichtyofauna; Lower Cretaceous; fluvial system.

Estudos paleontológicos realizados durante recente trabalho de mapeamento da CPRM - Serviço Geológico do Brasil na Sub-bacia de Tucano Central revelaram a ocorrência de materiais osteológicos de peixes na Formação São Sebastiāo. A biota dessa formação é pouco estudada e sua ictiofauna desconhecida (Maisey 2000).

O objetivo deste estudo é apresentar e descrever o primeiro registro formal de peixes fósseis na Formação São Sebastião, na porção pertencente à Sub-bacia de Tucano Central. Como as espécies identificadas como Lepidotes encontradas no Brasil não foram revisadas nem posicionadas em outro grupo até o presente momento, convém nomear as espécies neste trabalho utilizando aspas duplas (“”).

\footnotetext{
Instituto de Biociências da Universidade Federal do Estado do Rio de Janeiro - UNIRIO, Rio de Janeiro (RJ),Brasil. E-mail: hanna.clp@gmail.com

${ }^{2}$ Divisão de Paleontologia do Departamento de Geologia, Serviço Geológico do Brasil - CPRM, - Rio de Janeiro (RJ), Brasil. E-mail: rafael.costa@cprm.gov.br ${ }^{3}$ Gerência de Recursos Minerais, Superintendência de Marcos Regulatórios/SA, Serviço Geológico do Brasil - CPRM, - Salvador (BA), Brasil. E-mail: carolina@cprm.gov.br e caroline.santos@cprm.gov.br

*Autor correspondente

Manuscrito ID 29966. Recebido em: 25/04/2013. Aprovado em: 19/09/2013.
} 


\section{MATERIAL E MÉTODOS}

Os exemplares procedem de um afloramento da Formação São Sebastião, Sub-bacia de Tucano Central, situado em uma encosta de morro (1001'53,62”S; 385630,12”O), próximo ao Município de Canudos, nordeste do Estado da Bahia (Fig. 1). As amostras foram preservadas em arenito e encontravam-se soltas no afloramento e, devido à densa cobertura vegetal, não foi possível estabelecer com precisão os níveis de onde procedem.

O material consiste em 6 amostras, contendo cerca de 100 escamas e ossos isolados, além de um exemplar em posição de articulação, porém fragmentado em 10 amostras e preservado em concreção ferrificada. O material está depositado na coleção de Paleontologia do Museu de Ciências da Terra, CPRM - Serviço Geológico do Brasil. Essas amostras foram preparadas mecanicamente com ponteiras e cinzéis de aço de vários tamanhos, para permitir a observação das características morfológicas. A descrição das escamas foi realizada considerando a presença ou a ausência de ornamentação no bordo posterior, espessura e presença ou ausência de ornamentação da camada de ganoína, com base em Gallo-da-Silva (1998) e Gallo e Brito (2004). As amostras foram também comparadas com exemplares da coleção paleontológica do Museu de Ciências da Terra (MCT) para melhor observação das características morfológicas.

\section{CONTEXTO GEOLÓGICO}

A Bacia de Tucano situa-se no Estado da Bahia e é dividida nas sub-bacias de Tucano Norte, Central e Sul. Segundo Costa et al. (2007), essa bacia apresenta sequências deposicionais que datam do Neojurássico ao Eocretáceo e ocupa uma área de aproximadamente $30.500 \mathrm{~km}^{2}$. Além disso, outros autores (Brito \& Campos 1982, Brandão 2004) argumentaram que o arcabouço estrutural relaciona-se ao processo de rifteamento, que resultou na fragmentação do Gondwana Ocidental e na abertura do Atlântico Sul durante o Eocretáceo.

A Formação São Sebastião data do Eocretáceo, do Berriasiano ao Aptiano, estendendo-se da Sub-bacia de Tucano Central à Bacia do Recôncavo (Costa et al. 2007). Essa unidade foi originada por ciclos deposicionais de assoreamento do rifte, com uma arquitetura retrogradacional (Brandão 2004) e é caracterizada por arenitos grossos, amarelo-avermelhados, com intercalaçóes de alto teor de argilitos sílticos, com estratificação cruzada e em estrutura acanalada de grande porte, apresentando paleocorrentes que indicam variadas direçôes de fluxo (Gama Jr. 1970, Rocha \& Amaral 2007).

$\mathrm{Na}$ base do afloramento, ocorrem arenitos arcoseanos finos, com mica branca e óxidos, e matriz argilosa, com estrutura sigmoidal e tabular, além de estratificação cruzada acanalada e plano-paralela incipiente. Essa camada é seguida

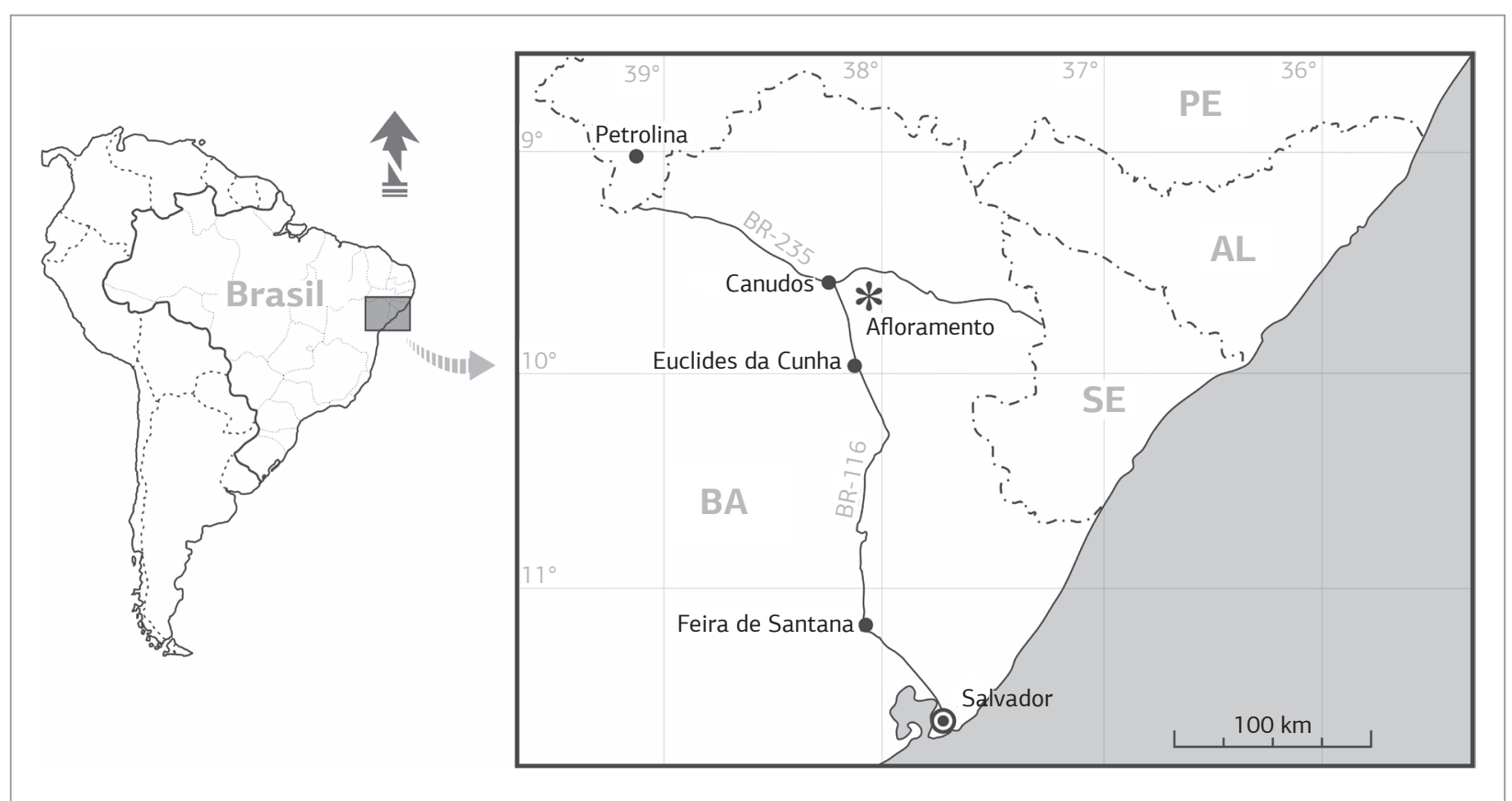

Figura 1. Mapa com a localização do afloramento na Formação São Sebastião, Sub-bacia de Tucano Central, Estado da Bahia. 
de folhelhos rosa acastanhados e verde acinzentados intercalados, com laminação plano-paralela ressaltada pela variação de cor, em um depósito com espessura entre um e dois metros. Acima, ocorre arenito fino com níveis fossilíferos e, em direçáo ao topo, os arenitos apresentam granocrescência ascendente, culminando com arenito conglomerático, onde ocorrem níveis fluidizados com convoluçóes.

Gama Jr. (1970) e Rocha e Amaral (2007) afirmaram que a ocorrência de estruturas cruzadas acanaladas é característica de sistemas nos quais as feiçóes fluviais propriamente ditas (e.g. canais, diques marginais, barras de meandro etc.) seriam responsáveis pelos clastos mais grossos, e os depósitos de inundação, pelos folhelhos e siltitos dessa unidade.

\section{SISTEMÁTICA}

\section{OSTEICHTHYES Huxley, 1880}

ACTINOPTERYGII Cope, 1887

NEOPTERYGII Regan, 1923

LEPISOSTEIFORMES Hay, 1929

\section{Lepidotes Agassiz, 1832}

\section{“Lepidotes" sp.}

\section{Material MCT 1440 a MCT 1449}

\section{Descriçâo}

Exemplar preservado originalmente em posição de articulação, porém fragmentado em 10 amostras (Fig. 2). Todas as amostras apresentam escamas do tipo ganoide e formatos variados, relacionadas à disposição no corpo do indivíduo: as escamas das amostras MCT 1443, MCT 1446 e MCT 1447 são retangulares e pertencentes à porção anterior; as amostras MCT 1444, MCT 1448 e MCT 1449 apresentam escamas rômbicas e pertencem às regióes dorsal e ventral. As escamas das amostras MCT 1440, MCT 1441 e MCT 1445 não foram identificadas quanto à sua posição. As amostras encontram-se preservadas em três dimensōes, mas não são preservados crânio nem cauda do peixe. É possível observar em todas as amostras a imbricação, a espessura e a orientação das escamas. Elas estáo quebradas e são observáveis os níveis de deposiçáo da placa basal em todas as amostras, exceto na MCT 1442, na qual a camada de ganoína é preservada e lisa, mas apresenta-se de cor esbranquiçada e está quebrada na extremidade posterior da escama. Ocorre também na amostra
MCT 1442 um osso alongado e cilíndrico, representando possivelmente um raio de nadadeira. Em todas as amostras as escamas apresentam borda posterior lisa.

\section{Observaçöes}

Oito espécies do gênero Lepidotes são conhecidas em rochas do Nordeste brasileiro: "Lepidotes" mawsoni Woodward, 1888; "Lepidotes" roxoi Santos, 1953 e "Lepidotes" oliveirai Santos, 1969, Cretáceo Inferior da Bacia do Recôncavo; "Lepidotes" souzai Woodward, 1908, Cretáceo Inferior da Bacia de Almada; "Lepidotes" piauhyensis Roxo \& Löfgren, 1936, Jurássico Superior da Bacia do Parnaíba; "Lepidotes" dixseptiensis Santos, 1963, Cretáceo Superior da Bacia Potiguar; "Lepidotes" alagoensis Gallo, 2000, Cretáceo Inferior da Bacia Sergipe-Alagoas; "Lepidotes" wenzae Brito \& Gallo, 2003, Cretáceo Inferior da Bacia do Araripe. Apesar de várias espécies compartilharem características das escamas, a comparaçấo foi feita entre as espécies encontradas em rochas do Cretáceo Inferior. As amostras analisadas possuem escamas com processos anteriores preservados em algumas delas, borda posterior lisa, sem ornamentação; a escama de MCT 1442 preservou a camada de ganoína, que se apresenta contínua na borda posterior e também sem ornamentação. "Lepidotes" mawsoni diferencia-se do material em estudo por apresentar escamas relativamente maiores e mais espessas, borda posterior ornamentada com sulcos e cristas bem evidenciados, formando leves denteaçóes, camada de ganoína descontínua e os processos anteriores relativamente maiores do que os do exemplar analisado. Também se diferencia de "Lepidotes" wenzae, que apresenta escamas de borda lisa e sem ornamentação, porém são relativamente mais delgadas que o exemplar em estudo. "Lepidotes" oliveirai, apesar de apresentar escamas de borda lisa e camada de ganoína contínua e sem ornamentação, possui escamas relativamente mais delgadas e processos anteriores mais curtos em relaçáo ao exemplar estudado. "Lepidotes" roxoi também se diferencia do exemplar em estudo por apresentar ornamentação em forma de sulcos e cristas no bordo posterior e escamas relativamente mais delgadas, além da camada de ganoína apresentar crenulaçôes e ser esparsa ou ausente na borda posterior. "Lepidotes" souzai apresenta diferenças pela ornamentação em forma de sulcos e cristas na borda posterior, além disso, a camada de ganoína não apresenta crenulaçôes, mas é descontínua e as escamas são relativamente mais delgadas que o exemplar em estudo. "Lepidotes" alagoensis possui as escamas sem ornamentação, mas a borda posterior tem denteaçóes irregulares, diferindo também do exemplar em estudo. Em resumo, as escamas em análise diferenciam-se de "Lepidotes" mawsoni, "Lepidotes" roxoi, "Lepidotes" alagoensis e "Lepidotes" souzai quanto à ornamentação da borda, espessura e descontinuidade da camada de ganoína. Diferenciam-se também de 


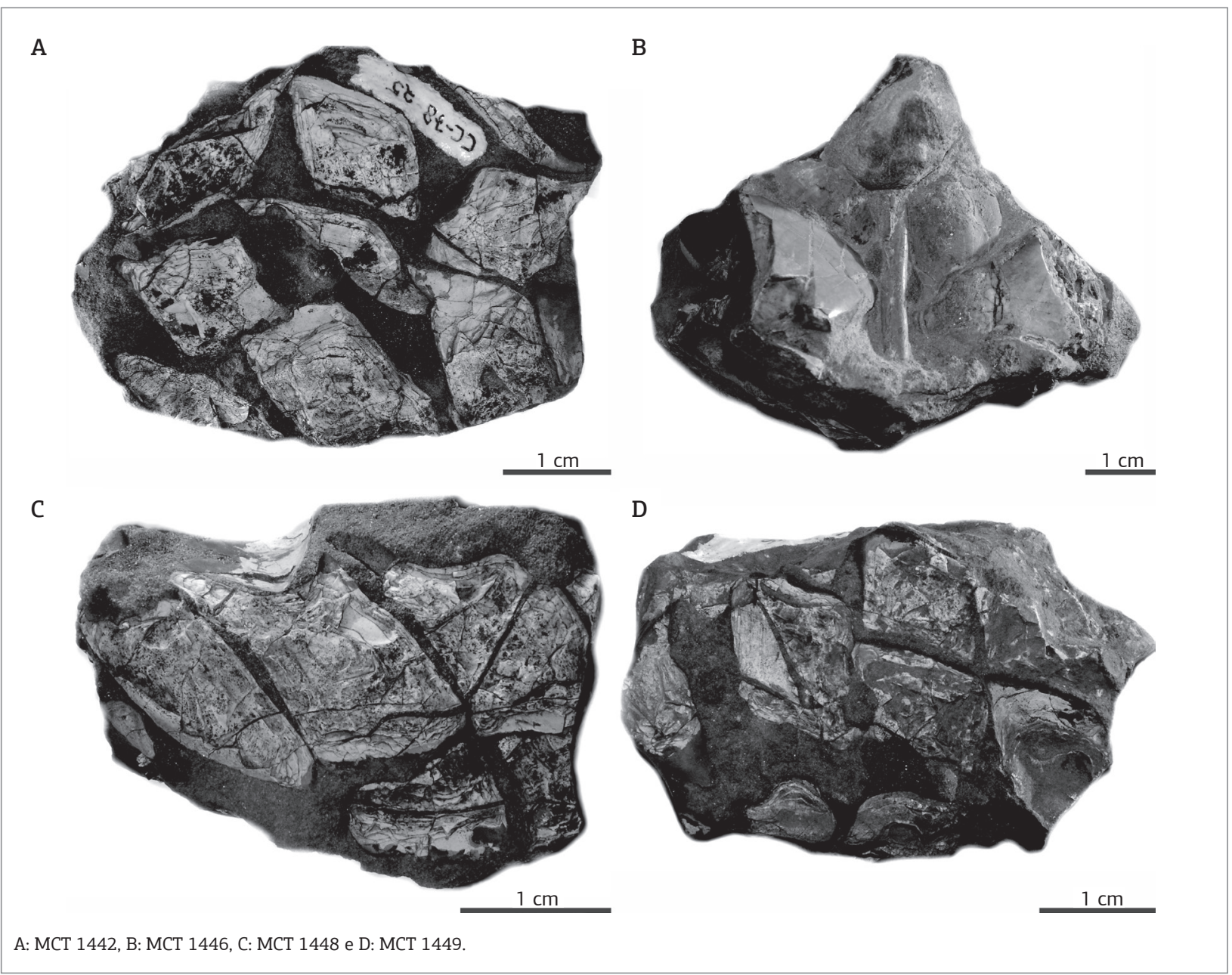

Figura 2. Amostras de "Lepidotes" sp. em vista lateral.

"Lepidotes" oliveirai e "Lepidotes" wenzae quanto à espessura e por apresentarem os processos anteriores mais curtos em relação ao exemplar estudado. Não é descartada a hipótese de que o exemplar em estudo corresponda a uma espécie nova, porém o material não apresenta características suficientes para uma descrição adequada. Devido à falta das características descritivas para uma nova espécie, mas se sabendo que o exemplar em estudo pertence ao gênero Lepidotes, sugere-se identificar o exemplar como "Lepidotes" sp.

\section{“Lepidotes” oliveirai Santos, 1969}

Peixe caracterizado por apresentar escamas muito delgadas, com excrescência interna muito reduzida, zona de imbricação muito curta, presença da articulação "peg-and-socket" nas escamas anteriores e medianas, apêndices anteriores muito curtos, camada de ganoína sobre a superfície exposta bastante regular e contínua, lisa, com borda posterior praticamente inteira e sem denteaçóes. Organização histológica lepisosteoide; camada de ganoína pouco espessa em relação à placa óssea basal (proporçáo 1:10); pouco numerosos, longos (por vezes, curtos) e não-ramificados canalículos de Williamson atravessando a placa basal, porém apenas raros ultrapassam o limite ganoína-placa basal; contato entre ganoína e placa basal plano no centro, passando a levemente serrilhado nas bordas da escama; numerosos osteócitos entre as lamelas ósseas; fibras de Sharpey muito densas e ramificadas (diagnose revista por Gallo-da-Silva 1998).

\section{Material MCT 1450}

\section{Descrição}

O exemplar consiste em uma escama ganoide, de formato rômbico, pertencente provavelmente à porção do flanco (Fig. 3F). A porção anterior da escama é mais delgada do que a posterior. A borda posterior é lisa e a camada de ganoína é preservada em apenas uma pequena parte da porção posterior da escama e não apresenta ornamentaçóes. Os dois processos anteriores (dorsal e ventral) foram preservados e são relativamente curtos. Possui excrescência ventral pouco intumescida e apresenta a articulaçáo do tipo "peg-and-socket". 


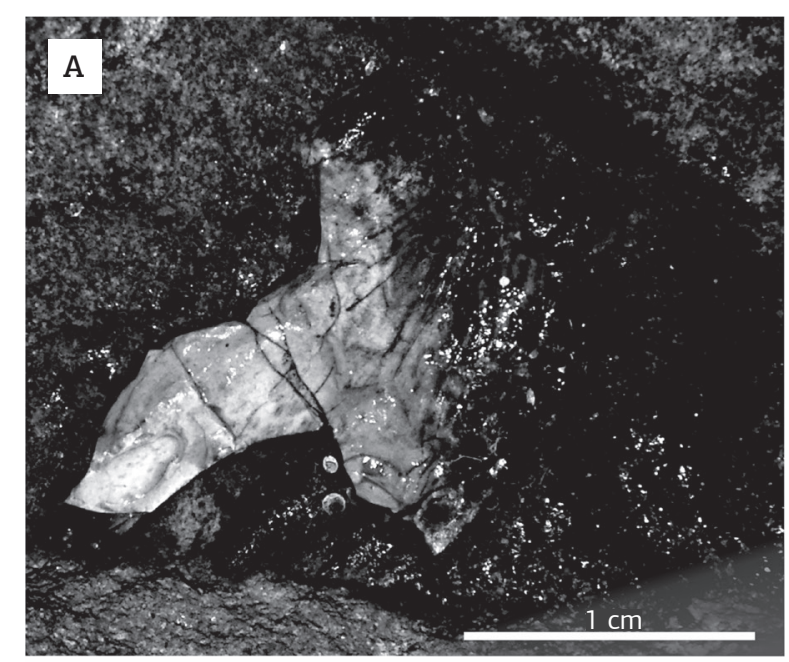

B

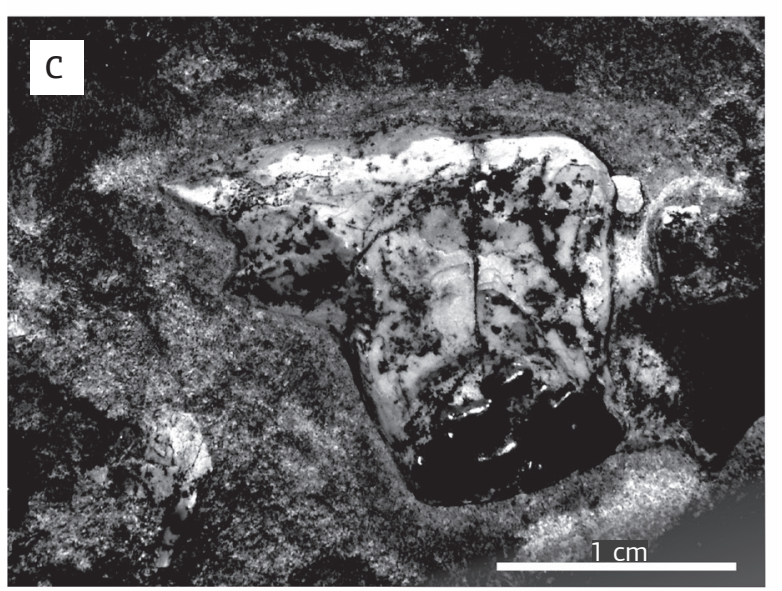

D
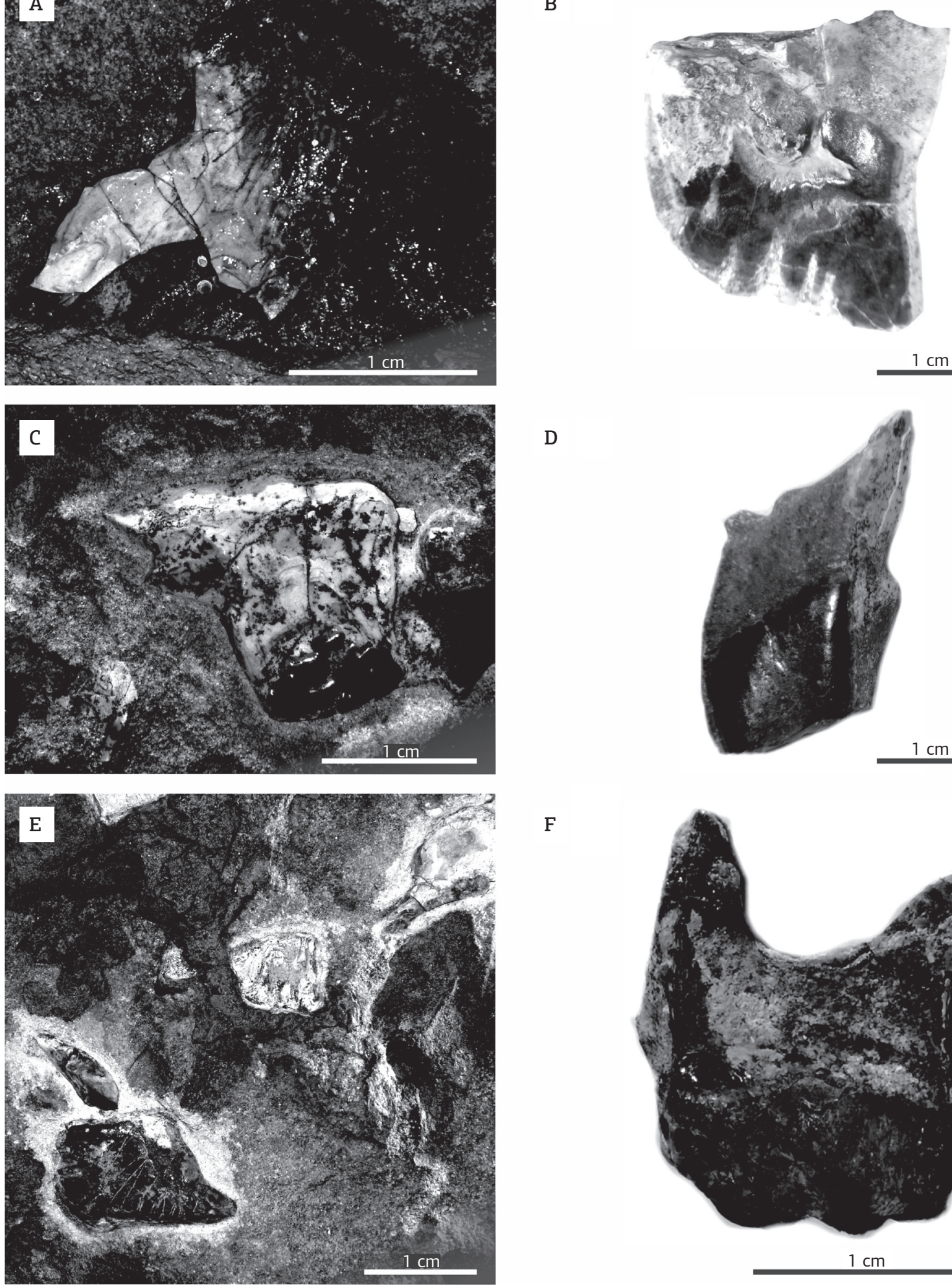

F
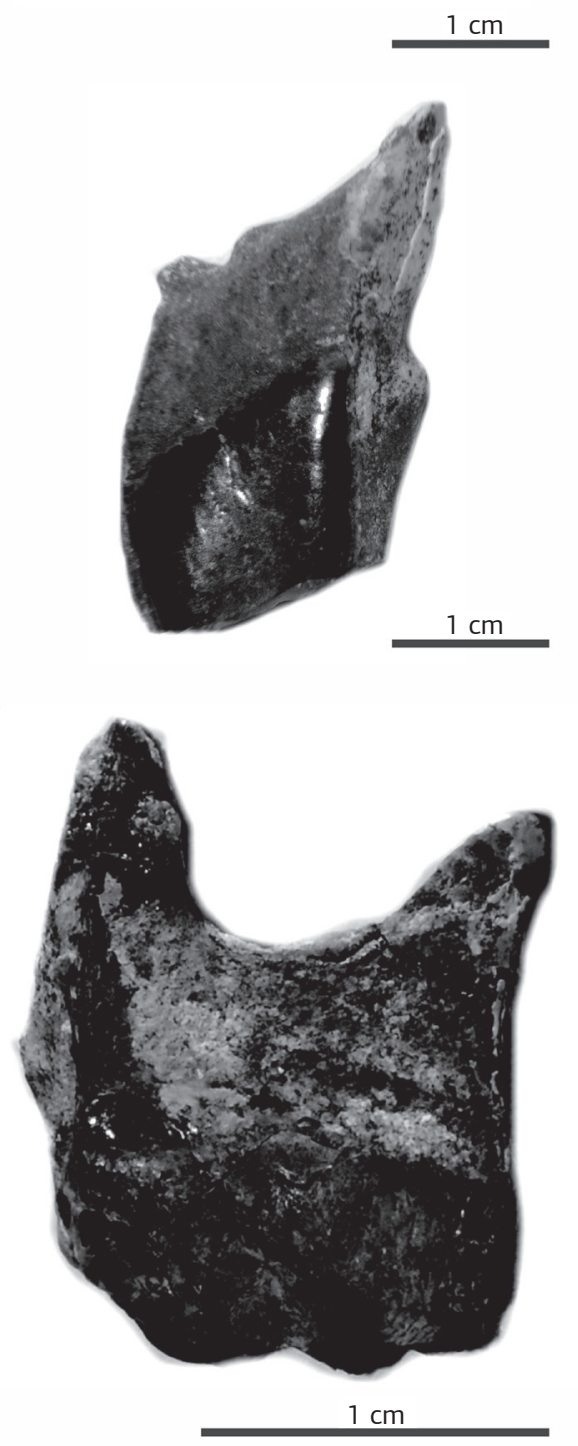

A: osso do crânio isolado de MCT 1454; B e D: escamas isoladas de MCT 1453; C: osso do crânio isolado de MCT 1451; E: escamas isoladas de MCT 1452 e F: escama de “Lepidotes" oliveirai, em vista lateral: MCT 1450.

Figura 3. Amostras de "Lepidotes" spp. em vista lateral. 


\section{Observaçóes}

As características do exemplar são comuns ao gênero Lepidotes. O tamanho dos processos anteriores e da excrescência ventral conferem com "Lepidotes" oliveirai, espécie baseada em escamas isoladas encontradas em rochas do Cretáceo Inferior da Bacia do Recôncavo. Pelo fato de a escama em estudo ser o único exemplar da espécie neste artigo, não foi possível a laminação. Sugere-se determinar o exemplar analisado como "Lepidotes" oliveirai, pela maior aproximação do exemplar estudado com a descrição feita anteriormente da metaespécie.

\section{“Lepidotes” spp.}

\section{Material MCT 1451 a MCT 1455}

\section{Descrição}

As amostras são, em maioria, associações de ossos e escamas isolados (Fig. 3A - 3E). Nas amostras MCT 1451 e MCT 1454, há prováveis ossos do crânio, mas em MCT 1451 o osso é ornamentado e com resquícios da camada de ganoína. Há também ossos fragmentados, desgastados, cilíndricos e alongados, porém sem identificação precisa na amostra MCT 1451. Todas as amostras apresentam escamas ganoides de diferentes morfologias: as escamas retangulares da porção anterior do corpo estão nas amostras MCT 1452 (uma escama inteira) e na amostra MCT 1453 (uma escama quebrada, mas identificável). A camada de ganoína foi preservada em todas as escamas das amostras MCT 1452 e MCT 1453. Esta é contínua e exibe ornamentação em forma de sulcos na porção posterior nas escamas das amostras MCT 1452 e MCT 1453. A porção anterior das escamas é mais delgada. Uma das escamas da amostra MCT 1453 possui o processo anterior (dorsal) preservado e duas escamas dessa mesma amostra apresentam a articulação "peg-and-socket"; a escama quebrada na porção posterior pertencia à região ventral, enquanto a outra escama, com borda posterior recortada, pertencia ao lado direito. Também é possível observar uma excrescência relativamente reduzida na porção ventral das escamas da amostra MCT 1453. Há também escamas desgastadas, porém com formato preservado nas amostras MCT 1455 (uma escama caudal). Algumas escamas da amostra MCT 1453 apresentam-se quebradas e não são identificáveis, mas ainda com a camada de ganoína presente.

\section{Observaçóes}

As escamas são de formatos e tamanhos variados. Em algumas, a borda posterior apresenta-se lisa e, em outras, ocorrem sulcos. Essas características são vistas em diferentes espécies de Lepidotes, assim as escamas não podem pertencer à mesma espécie. Os ossos não apresentam características diagnósticas para uma análise mais aprofundada. Sugere-se, então, determinar esses exemplares como "Lepidotes" spp., por conta de sua diferença em relação às demais amostras acima descritas.

\section{DISCUSSÃO}

Santos e Carvalho (2009) afirmaram que os peixes do gênero Lepidotes apresentavam características morfológicas típicas, como uma resistente armadura de ossos e escamas cobertos por ganoína, que os protegia contra predadores. A boca com dentes pequenos e de cúspides achatadas, tipicamente encontrada em indivíduos completos descritos para esse gênero, indica que eles se alimentavam provavelmente de pequenos peixes, crustáceos e moluscos, ou de vegetação de fundo nos lagos rasos (Gallo-da-Silva 1998, Galloda-Silva \& Azevedo 1998, Santos \& Carvalho 2009). De acordo com as características morfológicas descritas para o gênero Lepidotes encontrado no Brasil, esses peixes eram lentos e algumas espécies, como as encontradas na Formação Pastos Bons (Bacia do Parnaíba), formaçôes Maracangalha e Candeias (Bacia do Recôncavo) e formações Bananeiras e Barra de Itiúba (Bacia de Sergipe-Alagoas), se habituaram às águas rasas e calmas dos ambientes conquistados, antes mesmo da abertura do Atlântico Sul (Gallo-da-Silva, 1998, Maisey 2000, Santos \& Carvalho 2009). Alguns autores, como Maisey (1991), Santos e Carvalho (2009) e Wenz (1999), afirmaram também que esse gênero tem anatomia conservadora, pois mudaram pouco ao longo da sua história evolutiva.

López-Arbarello (2012) realizou uma análise filogenética de Ginglymodi viventes e fósseis e concluiu que o gênero Lepidotes deve ser revisto, pois algumas espécies atribuídas ao gênero provavelmente pertenceriam a outros grupos. Como não há revisão das espécies de Lepidotes no momento, convém nomear os fósseis estudados nesse trabalho como pertencentes ao gênero Lepidotes.

De acordo com Holz e Simôes (2002), os sistemas fluviais apresentam constante renovação hídrica e os animais mortos neles tendem a ser transportados e desarticulados após a morte, seguindo o fluxo das correntes. Outros autores, como Elder e Smith (1984), afirmaram ainda que o processo de transporte, desarticulação e fragmentaçáo de ossos ou escamas pode variar de intensidade dependendo do peso do esqueleto ou da atividade de necrófagos antes do soterramento.

O gênero Lepidotes é mais conhecido no Brasil pela presença abundante de escamas nas bacias sedimentares, as quais geralmente são mais resistentes ao transporte hídrico por serem elementos numerosos, densos e mais resistentes em 
relação aos ossos (Santos \& Carvalho 2009, Pinheiro et al. 2011). Devido à constante renovação hídrica dos sistemas fluviais, o registro de um espécime articulado é dificilmente citado na literatura, pois geralmente fósseis de peixes são melhor preservados em ambientes marinhos e lacustres (Gallo et al. 2011).

No caso do exemplar identificado como "Lepidotes" sp., provavelmente o indivíduo sofreu pouca necrólise, pouco ou nenhum transporte e o soterramento foi relativamente rápido, preservando assim as escamas em sua posição original de articulação. O material foi preservado na forma de uma concreção ferrificada, porém o exemplar foi danificado pelo intemperismo anterior à coleta, que removeu suas camadas mais exteriores. Assim, encontra-se exposta a camada óssea abaixo da porção ganoide, que foi quase completamente destruída. As escamas não foram perdidas, mas a camada de ganoína foi encontrada apenas no exemplar MCT 1442. Além do raio de nadadeira, não foram encontrados outros ossos. Provavelmente a camada de ganoína seguia o mesmo padrão do exemplar MCT 1442 no peixe inteiro.

A ocorrência de escamas e ossos desarticulados indica que os outros exemplares analisados sofreram necrólise, desarticulação e transporte em variados graus antes de serem soterrados. Os ossos e escamas apresentam mais ou menos o mesmo tamanho e algumas escamas preservaram parcialmente a camada de ganoína. Autores como Elder e Smith (1984, 1988), Holz e Simóes (2002) e Chellouche et al. (2012) afirmaram que o fato de escamas e ossos serem encontrados juntos indica que a corrente hídrica do afloramento tinha seleção moderada, pois transportava tanto elementos mais densos (as escamas) como elementos menos densos (os ossos).

O fato de encontrar espécimes articulados associados a outros fragmentados e desarticulados permite sugerir que a associação fossilífera seria parautóctone ou mais provavelmente uma mistura de elementos autóctones e alóctones (Holz \& Simôes 2002).

\section{CONCLUSÃO}

Até o momento, o registro formal de fósseis de peixes da Formação São Sebastião, na porção pertencente à Subbacia de Tucano Central, era desconhecido, sendo esse o primeiro registro da ictiofauna - eles foram identificados como "Lepidotes" sp., "Lepidotes" oliveirai e "Lepidotes" spp. (Actinopterygii, Lepisosteiformes).

Os atuais resultados permitem ampliar o conhecimento paleontológico sobre as biotas cretácicas do Nordeste e revelam o potencial da região para a pesquisa científica.

\section{AGRADECIMENTOS}

À Dra. Valéria Gallo, da Universidade do Estado do Rio de Janeiro, pelo inestimável apoio na identificação dos exemplares estudados, à Dra. Marise Sardenberg Salgado de Carvalho e à Dra. Márcia Aparecida dos Reis Polck, do Departamento Nacional de Produção Mineral, pelo apoio na identificação do material e ao Rodrigo da Rocha Machado pelo acesso à coleção de Paleontologia do Museu de Ciências da Terra. HCLP agradece à CPRM - Serviço Geológico do Brasil pela bolsa concedida. RCS agradece ao Conselho Nacional de Desenvolvimento Científico e Tecnológico (CNPq) pela bolsa de produtividade. O apoio financeiro foi concedido pela CPRM - Serviço Geológico do Brasil.

\section{REFERENCES}

Agassiz L.R. 1832. Untersuchungen über die fossilen Fische der LiasFormation. Neues Jahrbuch für Mineralogie, Geognosie. Geologie und Petrefaktenkunde, 3:139-149.

Brandão H.C. 2004. Interpretação conjunta de dados de GPR e medidas de permeabilidade sobre um análogo de reservatório siliciclástico falhado na Bacia do Tucano, NE do Brasil. MS Dissertation, Programa de Pós Graduação em Geologia, Universidade Federal do Rio Grande do Norte, Natal, 106 p.

Brito I.M. \& Campos D.A. 1982. O Cretáceo no Brasil. In: $54^{\circ}$ Congresso Brasileiro de Paleontologia, Anais da Academia Brasileira de Ciências, p. 197-218.

Brito P. \& Gallo V. 2003a. Presença de Lepidotes Agassiz, 1832 (Actinopterygii, Semionotidae) na Formação Santana, Cretáceo Inferior da Bacia do Araripe, Nordeste do Brasil. Boletim do Museu Nacional, 67:1-7.
Brito P. \& Gallo V. 2003b. A new species of Lepidotes (Neopterygii: Semionotiformes: Semionotidae) from the Santana Formation, Lower Cretaceous of Northeastern Brazil. Journal of Vertebrate Paleontology, 23(1):47-53.

Chellouche P., Fürsich F.T., Mäuser M. 2012. Taphonomy of neopterygian fishes from the Upper Kimmeridgian Wattendorf Plattenkalk of Southern Germany. Palaeobiodiversity and Palaeoenvironments, 92(1):99-117.

Cope E.D. 1887. Observations on the systematic relations of the fishes. Proceedings of the American Association for the Advancement of Science, 20:317-343.

Costa I.P., Milhomem P.S., Buenos G.V., Silva H.S.R.L., Kosin M.D. 2007. Sub bacias de Tucano Sul e Central. Boletim de Geociências da Petrobrás, 15(2):433- 443. 
Elder R.L. \& Smith G.R. 1984. Fish taphonomy and paleoecology. Geobios, 17(1):287-291.

Elder R.L. \& Smith G.R. 1988. Fish taphonomy and environmental inference in paleolimnology. Palaeogeography, Palaeoclimatology, Palaeoecology, 62:577-592.

Gallo V. 2000. First occurrence of Lepidotes (Actinopterygii: Semionotiformes: Semionotidae) in the Coqueiro Seco Formation, Early Aptian of the Sergipe-Alagoas basin, northeastern Brazil. Boletim do Museu Nacional, Nova Série Geologia, 51:1-14.

Gallo V. 2005. Redescription of Lepidotes Piauhyensis Roxo and Löfgren, 1936 (Neopterygii, Semionotiformes, Semionotidae) from the Late Jurassic-Early Cretaceous of Brazil. Journal of Vertebrate Paleontology, 25(4):757-769.

GalloV.\&Brito P.M. 2004. An overview of Brazilian semionotids. In: G. Arratia \& A. Tintori (eds.), Mesozoic Fishes 3 - Systematics, Paleoenvironments and Biodiversity, Verlag Dr. Friedrich Pfeil, München, p. 253-264.

Gallo V., Calvo J. \& Kellner A.W.A. 2011. First record of a clupeomorph fish in the Neuquén Group (Portezuelo Formation), Upper Cretaceous of Patagonia, Argentina. Cretaceous Research, 32:223-235.

Gallo-da-Silva V. 1998. Revisão das espécies do gênero Lepidotes Agassiz, 1832 (Actinopterygii, Semionotiformes) do Mesozóico do Brasil, com comentários sobre as relações filogenéticas da família Semionotidae. PhD Thesis, Instituto de Geociências, Universidade de São Paulo, São Paulo, 259 p.

Gallo-da-Silva V. \& Azevedo S.A. 1998. Histo-morphological study of Lepidotes teeth (Neopterygii: Semionotidae) from the Lower Cretaceous of Brazil. Anais da Academia Brasileira de Ciências, 70(3): 441-452.

Gama Jr. E. 1970. Modelo geológico das bacias de Recôncavo e Tucano. In: $24^{\circ}$ Congresso Brasileiro de Geologia da Sociedade Brasileira de Geologia, Anais, p. 191-200.

Hay O.P. 1929. A collection of Pleistocene vertebrates from southwestern Texas. Proceedings of the United States National Museum, 68:1-18.

Holz M. \& Simões M.G. (eds.) 2002. Elementos fundamentais de Tafonomia. Porto Alegre, UFRGS, 240 p.

Huxley T.H. 1880. On the applications of the laws of evolution to the arrangement of the Vertebrata. Proceedings of the Zoological Society of London, 649-662.

López-Arbarello A. 2012. Phylogenetic Interrelationships of Ginglymodian Fishes (Actinopterygii: Neopterygii). PLOS ONE, 7(7): 1-44.

Maisey J.G. (ed.) 1991. Santana Fossils: An Illustrated Atlas. Neptune City, T.F.H. Publications, $462 \mathrm{p}$.
Maisey J.G. 2000. Continental break up and the distribution of fishes of Western Gondwana during then Early Cretaceous. Cretaceous Research, 21:281-314.

Pinheiro F.L., Figueiredo A.E.Q., Fortier D.C., Viana M.S.S., Schultz C.L. 2011. Fauna de Vertebrados Eocretácicos de um afloramento da Bacia de Lima Campos, Ceará, Brasil. Revista Brasileira de Paleontologia, 14(2):189-198.

Regan C.T. 1923. The skeleton of Lepisosteus, with remarks on the origin and evolution of the lower Neopterygian fishes. Proceedings of the Zoological Society of London, 445-461.

Rocha D.E.G.A. \& Amaral C.A. 2007. Comportamento das bacias sedimentares da região semiárida do nordeste brasileiro, Meta B - Caracterização Geológica e Geométrica dos Aquíferos. Rede Cooperativa de Pesquisa CPRM - Serviço Geológico do Brasil e UFPE Universidade Federal de Pernambuco. 35 p.

Roxo M. \& Löfgren A. 1936. Lepidotus piauhyensis, sp. nov. Notas Preliminares e Estudos, DGM/ DNPM, 1:7-12.

Santos M.E.C.M. \& Carvalho M.S.S. 2009. Paleontologia das bacias do Parnaíba, Grajaú e São Luís -Reconstituições Paleobiológicas. CPRM Serviço Geológico do Brasil. 193 p.

Santos R.S. 1953. Peixes triássicos da Fazenda Muzinho, Estado do Piauí. Notas Preliminares e Estudos, DGM/ DNPM, 70:1-4.

Santos R.S. 1963. Peixes do Cretácico do Rio Grande do Norte. In: $35^{\circ}$ Congresso Brasileiro de Paleontologia, Anais da Academia Brasileira de Ciências, 1:67-74.

Santos R.S. 1969. Sobre um Lepidotes da Formação Itaparica, Estado da Bahia. Boletim de Geologia do Instituto de Geociências da UFRJ, 4:43-46.

Wenz S. 1999. Pliodetes nigeriensis gen. et. sp. nov., a new semionotid fish from the Lower Cretaceous of Gagoufaua (Niger Republic): Phylogenetic comments. In: Arratia G. \& Schultze H.P. (eds): Mesozoic Fishes 2 - Systematics and Fossil Record: 107-120.

Woodward A.S. 1888. Notes on some vertebrate fossils from the Province of Bahia, Brazil, collected by Joseph Mawson. Annals and Magazine of Natural History, 2(8):132-136.

Woodward A.S. 1908. On some fossil fishes discovered by Prof. Ennes de Souza in the Cretaceous Formation at Ilheus (State of Bahia), Brazil. Quartertly Journal of Geological Society, 64:358-362.

Arquivo digital disponível on-line no site www.sbgeo.org.br 